\title{
Improved Spin-orbit Torque Induced Magnetization Switching Efficiency by Helium Ion Irradiation
}

\author{
Suhyeok An \\ DGIST \\ Eunchong Baek \\ DGIST \\ Jin-A Kim \\ DGIST \\ Ki-Seung Lee \\ DGIST \\ Chun-Yeol You ( $\nabla$ cyyou@dgist.ac.kr) \\ DGIST
}

\section{Research Article}

Keywords: spin-orbit torque (SOT), magnetization switching efficiency, Helium ion irradiation

Posted Date: October 25th, 2021

DOl: https://doi.org/10.21203/rs.3.rs-997332/v1

License: (c) (1) This work is licensed under a Creative Commons Attribution 4.0 International License.

Read Full License

Version of Record: A version of this preprint was published at Scientific Reports on March 2 nd, 2022. See the published version at https://doi.org/10.1038/s41598-022-06960-8. 


\section{Abstract}

Increasing the efficiency of spin-orbit torque (SOT) is of great interest in spintronics devices because of its application to the non-volatile magnetic random access memory and in-logic memory devices. Accordingly, there are several studies to alter the magnetic properties and reduce the SOT switching current with Helium ion irradiation, but previous researches are focused on its quantitative changes only. Here, the authors observe the reduction of switching current and analyze the origins of Helium ion irradiation induced SOT switching current reduction. The first is from improved spin Hall angle caused by the resistivity change of heavy metal layer and second is from the reduction of surface anisotropy energy at interface between heavy metal and ferromagnet. The result shows that switching current is reduced about $\sim 30.3 \%$ at dose of 30 ions $/ \mathrm{nm}^{2}$ and relevant parameter change is shown as improved spin Hall angle from 0.096 to 0.132 and reduced anisotropy field from 13.7 to $8.5 \mathrm{kOe}$. Altogether, the power consumption ratio is calculated based on the derived parameter and result shows that requiring power reaches only $56.0 \%$ at 30 ions $/ \mathrm{nm}^{2}$. This analysis suggests that more efficient SOT device engineering is possible by Helium ion irradiation.

\section{Introduction}

Spin-orbit torque (SOT) induced magnetization switching is a perspective phenomenon to magnetic material based devices because of its potential application to the non-volatile magnetic random access memory (MRAM) and in-logic memory devices. SOT devices have two main benefits compare to the spin transfer torque (STT) MRAM. First, SOT switching is much faster ( $1 \mathrm{~ns})$ than STT-MRAM ( $10 \mathrm{~ns})$. Second, SOT-MRAM have reading path separated from the writing path, it is expected that more stable devices are possible and more margins in the reading and writing currents. In SOT switching, there are two essential ingredients: structural inversion symmetry breaking and strong spin-orbit coupling (SOC). A heavy metal (HM) / ferromagnetic metal (FM) bilayer structure satisfies those requirements. When charge current passes through the $\mathrm{HM}$, the spin current is created inside of $\mathrm{HM}$ and injected into the FM by spin Hall effect (SHE) ${ }^{1}$ and/or the Rashba effect can create non-zero effective field at the interfaces ${ }^{2}$. For developing more effective SOT induced magnetization switching, many relevant parameters like spin Hall angle (SHA, $\left.\theta_{S H}\right)^{3}$, magnetic anisotropy field ${ }^{4}$ and Dzyaloshinskii-Moriya (DM) interaction ${ }^{5}$ are under the investigation. Among them, $\theta_{S H}$, a ratio of spin current to the charge current densities, is a vital parameter as indicators of converting efficiency from electric charge to spin current densities by SHE in HM. The $\theta_{S H}$ is one of the key players on SOT induced switching since the magnitude of SOT is proportional to the spin current. Therefore, $\theta_{S H}$ value has been investigated heavily in various $\mathrm{HMs}$, like Ta $(\sim 0.15)^{6}, \beta-\mathrm{W}$ $(\sim 0.33)^{7}$ and $\mathrm{Pt}(\sim 0.1)^{8,9}$.

Since the discovery of SOT, intensive efforts to enhance and control the strength of SOT are conducted by annealing ${ }^{10}$, resistivity control of HM layer ${ }^{11}$, normal metal (NM) insertion ${ }^{12,13}$, alloying ${ }^{14}$, interface modifying ${ }^{15,16}$, and ion irradiation ${ }^{17,18}$. Among them, the method of irradiating Helium ion is known as leading a structural rearrangement while maintaining the overall atomic layer districts in the multi- 
stacked structure ${ }^{19}$ even if no ions remain in the sample due to the long penetration depth $(>50 \mathrm{~nm})^{20}$. Ion irradiation induced structural reorganization affects layers and interfaces, resulting in the coercivity and anisotropy field change ${ }^{21}$, domain wall (DW) pinning site creation ${ }^{22}$, modulating DM Interaction ${ }^{23}$, and performing magnetic skyrmions ${ }^{24}$. This characteristic is also evident in the case of SOT, so it has been observed in various SOT relevant effects such as reduction of switching current by SOT ${ }^{18}$, influence on DW dynamics ${ }^{25}$, and multi-level state ${ }^{26}$. However, none of the previous studies about Helium ion irradiation modifying SOT phenomena have been paid attention in analyzing its origins by separating the properties of HM and FM layer.

Here, we successfully distinguished the effects of ion irradiations on HM/FM bilayer system, as changes of $\theta_{S H}$ and PMA strength in $\mathrm{HM}$ and $\mathrm{FM}$ layer. We prepared $\mathrm{Pt}(5) / \mathrm{Co}(0.8) / \mathrm{MgO}(2)$ structure samples and irradiated Helium ions in various doses. After Helium ions irradiations, we measured the critical current for SOT induced switching with various in-plane field. We found that the switching current decreases from 16.1 to $11.2 \mathrm{~mA}$ as increasing dose amounts from 0 to 30 ions $/ \mathrm{nm}^{2}$ with the in-plane field of 3.1 kOe. For understanding the decrease of switching current, we also measured the first and second order uniaxial anisotropy fields, and $\theta_{S H}$ determined by the harmonic Hall measurement method. By careful analysis including the second order uniaxial anisotropy field contributions, we extracted magnetization polar angular dependent effective field caused by SOT for each dose and $\theta_{S H}$ from $\Delta H_{D L}$. Furthermore, we measured the effect of the dose amount on the resistivities of single Pt layers and confirmed the relation between resistivity and $\theta_{S H}$. Based on our analysis, the reduction of the switching current partially ascribes to the reduced anisotropy field from 13.7 to $8.5 \mathrm{kOe}(38.0 \%)$ and improved $\theta_{S H}$ from 0.096 to $0.132(27.2 \%)$. Here, it must be mentioned that the reduction rate of switching current $(30.4 \%)$ is smaller than expected reduction rate when we consider the magnetic anisotropy reduction and $\theta_{S H}$ enhancement. Nevertheless, the calculated power consumption was $87.4 \%, 59.6 \%$, and $56.0 \%$ at each dose compared with the non-irradiated sample, indicating that Helium ion irradiation can contribute to better SOT device fabrication.

\section{Results}

We deposited $\mathrm{Pt}(5 \mathrm{~nm}) / \mathrm{Co}(0.8 \mathrm{~nm}) / \mathrm{MgO}(2 \mathrm{~nm})$ heterostructure sample using magnetron sputter and patterned as $10 \mu \mathrm{m}$ width Hall bar structure by photolithography technique. In Figure 1a, the sample structure, coordinate systems, and Helium ion irradiation area are depicted. $\theta_{M}$ is the polar angle between magnetization direction and z-axis, $\theta_{B}$ is the polar angle between the external magnetic field and z-axis, and $\phi$ is azimuthal angle from $x$-axis, and here we ignore the angle differences between the external magnetic field and magnetization directions in the azimuthal angle because of the negligible in-plane anisotropy. After Hall bar fabrication process, Helium ion is irradiated in vertical direction with sample plane having acceleration energy of $30 \mathrm{keV}$, beam current of $5.5 \mathrm{pA}$, and dose from 0 to $30 \mathrm{ions} / \mathrm{nm}^{2}$ with step of 10 ions $/ \mathrm{nm}^{2}$. Irradiation area covers whole Hall cross for avoiding signal mixing errors by signal 
differences between irradiation and non-irradiation area as shown red dotted rectangle in Figure 1a (more explanation in Supplementary Figure S1).

Measurement of SOT induced magnetization switching includes three sequences: initialization, SOT writing by pulse current, and reading from Hall resistance measurement. Firstly, sample is under strong enough $+z$-axis (-z-axis) direction external field to saturate the magnetization in up (down) direction as initial state. After initialized, the current pulse is injected with pulse amplitude $(I p)$ from -25 to $25 \mathrm{~mA}$ ( 25 to $-25 \mathrm{~mA}$ ) with $1 \mathrm{~mA}$ step. During the current pulse injection in $\mathrm{x}$-axis direction in-plane magnetic field is applied to ensure deterministic switching. And the Hall resistance $\left(R_{H}\right)$ is measured in the middle of each pulse injection with $100 \mu \mathrm{A}$ magnitude direct current (DC). Here, we should mention that the $R_{H}$ is unit value calculated from measured Hall voltage $\left(V_{H}\right)$ dividing with magnitude of reading current. The $R_{H}$ results as a function of Ip with the previous procedure shows typical hysteresis loops, as shown in Figure 1b-1c, and it indicates SOT induced magnetization switching in PMA system. Here, the shown hysteresis loops in Figure $1 \mathrm{~b}$ are in case for dose amount of $30 \mathrm{ions} / \mathrm{nm}^{2}$. It is well known that higher in-plane field make switching more easily, but we observe that Helium ion irradiation also reduces the switching current. The SOT driven magnetization switching hysteresis loops for various doses with a fixed $2.2 \mathrm{kOe}$ external in-plane field is shown in Figure 1c. In Figure 1d, the switching currents from magnetization switching loops at each dose and external magnetic field are depicted. In Figure 1d, we found two features. Firstly, in small field region $(0.7 \mathrm{kOe})$, rapid increase of switching current appears. We expect that this increase is caused by the nucleation of multi-domain states during SOT induced magnetization switching process (see the Supplementary Figure S2a for more details). Because of the multi-domains under small field, it is hard to compare the switching current in higher field region directly so that we will not pay attention much. The second feature is main finding of this work in larger field regions $(\geq 1.2 \mathrm{kOe})$. We found that the switching current is reduced by increasing dose amounts. And at same dose amount, the switching current have linear relation with the external in-plane field strength under the sufficiently smaller in-plane field comparing 1 st order effective anisotropy field, which is well-known behavior following $J_{C}=\frac{2 e}{\hbar} \frac{M_{S} t_{F}}{\theta_{S H}}\left(\frac{H_{K, \text { eff }}}{2}-\frac{H_{X}}{\sqrt{2}}\right){ }^{28}$. And here, e is charge of electron, $\hbar$ is Planck constant, $M_{s}$ is saturation magnetization, $t_{F}$ is thickness of FM layer, $H_{K, \text { eff }}$ is the 1 st order anisotropy field and $H_{x}$ is inplane external magnetic field parallel with current. In order to get better insight of the Helium irradiations effect, we show the switching current reduction ratio at each dose compared with the pristine sample ( $\left.\left|\left(I_{P . \text { crit }}-I_{P, \text { crit }}^{\text {Dose }}\right) / I_{P, \text { crit }}^{\text {Dose }}\right| \times 100 \%\right)$ as in Figure 1 e. Result shows that the switching current reduction has increasing tendency with dose amount for in-plane external field. The exceptional dependence for small field $(0.7 \mathrm{kOe})$ probably ascribe to the formation of multi-domain state as seen in Supplementary Figure S2b. The reduction ratio appears largely at external field of $3.1 \mathrm{kOe}$ about $14.2 \%, 25.5 \%$, and $30.3 \%$ at dose of 10,20 , and 30 ions $/ \mathrm{nm}^{2}$, respectively. Here, the possible physical origins of the switching current reduction can be the enhanced $\theta_{S H}$ of Pt layers, and/or it can be the decrease of the effective anisotropy field of FM layer. We will discuss more details later. 
To understand the more details of switching behavior, the effects of Helium ion irradiation on the magnetic anisotropy fields are investigated. We conducted Anomalous Hall effect (AHE) measurement by swapping the external magnetic field in z-axis direction to obtained normalized Hall resistance $\left(R_{H}\left(H_{e x t}\right) / R_{H}\left(H_{e x t}=0\right.\right.$ Oe $\left.)\right)$ hysteresis loops, because the AHE signal is proportional to the z-component of magnetization. The normalized AHE hysteresis loops in Figure 2a shows strong enough PMA for all samples. And each coercivity is decreasing ( $34 \%)$ from 271 Oe to 178 Oe as shown in Figure $2 b$ by increasing dose amounts. In order to obtain the 1 st and 2 nd order anisotropy fields $\left(H_{K, \text { eff }} H_{K, 2}\right)$ by using generalized Sucksmith-Thompson (GST) method (see Supplementary Figure S3), we measured normalized AHE by applying in-plane field $\left(H_{x}\right)$ along the current direction as seen in Figure 2c. Here, it must be mentioned that the obtained 1st order anisotropy fields are the effective anisotropy including demagnetization effect, not pure anisotropy field in GST method. The $H_{K, \text { eff }}$ and $H_{K, 2}$ are shown in Figure $2 \mathrm{~d}$ as a function of dose. By increasing dose amount from 0 to $30 \mathrm{ions} / \mathrm{nm}^{2}, H_{K, \text { eff }}$ and $H_{K, 2}$ decrease $38.2 \%$, and $27.5 \%$, respectively. We speculate that decrease is mainly caused by interface modulation from Helium ion irradiation process, since the surface anisotropy energy is very sensitive on the quality of the interface between HM and FM layers. Although it is hard to classify and/or probe the effect of the structural modulation caused by the Helium ion irradiation, we can claim that he anisotropic field as well as the coercivity field can be reduced by the Helium ion irradiation. The magnitude of $H_{K, 2}$ is only less than half (40.1\%) compared with the $H_{K, \text { eff }}$ however, the SOT analysis without consideration of $H_{K, 2}$ may lead incorrect results ${ }^{29}$.

Not only the anisotropy characteristics, but also an important parameter in SOT induced magnetization reversal is $\theta_{S H}$. The harmonic Hall signal analysis is frequently used method for calculating $\theta_{S H}$ as well as extracting SOT driven effective fields ${ }^{29,30}$. It is well known that the SOT has two contributions acting on different directions, so called field-like torque $\left(F L T, \Delta H_{F L}\right)$ in transverse direction and damping-like torque (DLT, $\Delta H_{D L}$ ) effective field in longitudinal direction, consideration of AHE and PHE resistances are necessary for obtaining correct results. In Figure 3a, the measured Hall resistance loops are shown at $\phi=$ 10 to $40^{\circ}$ with fixed $\theta_{B}=80^{\circ}$ for dose amount of 30 ions $/ \mathrm{nm}^{2}$ sample. Since the AHE and PHE contribute to the measured Hall signal as following the equation ${ }^{30}$,

$$
V_{H}=I_{0} R_{0}=\frac{I_{0} R_{A H E}}{2} \cos \theta_{M}+\frac{I_{0} R_{P H E}}{2} \sin ^{2} \theta_{M} \sin 2 \varphi
$$

1

The clear asymmetries are observed for the Hall loops in Figure 3a in the large field. The asymmetry also increases because of the larger PHE contribution for large $\phi$. And by adding and subtracting divided asymmetric Hall loop between $+B$ to $-B$ part and $-B$ to $+B$ part, one can separate the contributions of $A H E$ and PHE as seen in Figure $3 \mathrm{~b}$ and Figure 3c, respectively. Details of extracting method for AHE and PHE resistances is explained in Supplementary Figure S4. Here, Figure $3 b$ and Figure $3 c$ show the data at the angle of $\theta_{B}=80^{\circ}$ and $\phi=40^{\circ}$ at each dose amount. The AHE resistance can be calculated using AHE 
contribution at $\theta_{B}=0^{\circ}$, corresponding to zero external in-plane field, and the PHE resistance can be also calculated taking linear plot on the PHE contribution from the slope of $\sin ^{2} \theta_{M^{*}}$ in Equation (1). From those measurement analyses, the calculated AHE and PHE resistances are shown in Figure $3 d$ together. $\mathrm{R}_{\mathrm{AHE}}$ increased from 1.09 to $1.20 \Omega(9.8 \%)$ with increasing dose amounts, while $\mathrm{R}_{\mathrm{PHE}}$ varied within the range of 0.37 to $0.34 \Omega$. Since the ratio of $R=R_{P H E} / R_{A H E}$ has an important role in analysis of the harmonic Hall measurement result, we calculated the ratio and it changes from 0.34 to 0.29 at dose of 0 and 30 ions $/ \mathrm{nm}^{2}$, as depicted in Figure $3 \mathrm{e}$. It must be mentioned that the variation of $\mathrm{R}$ with Helium ion irradiation is not significant comparing to other physical quantities. One possible explanation is that the bulk magnetic properties are relatively insensitive on the Helium ion irradiation, while the surface properties, such as surface anisotropy, are more sensitive.

To obtain the $\theta_{S H}$ or SOT induced effective fields, we measure harmonic Hall with alternating current (AC) of $5.5 \mathrm{~mA}$ peak amplitude and $401 \mathrm{~Hz}$ frequency $\left(I_{A C}=I_{0} \sin 2 \pi f t\right)$. Because the harmonic Hall measurement is influenced by the Joule heating effect caused by current flow, we follow the fourdirection method for eliminating some thermoelectric artifacts ${ }^{31}$. The 1 st and 2 nd harmonic Hall loop is measured swapping magnetic field with fixed $\theta_{B}=85^{\circ}$ and $\phi=0^{\circ}$ for $\Delta H_{D L}$ and $\phi=90^{\circ}$ for $\Delta H_{F L}$ measurements. Each Hall loop result is shown in Figure 4a-4c, respectively. Here, the 1 st and 2 nd harmonics are measured simultaneously with two lock-in amplifiers at each $\phi$ and dose amounts. Harmonic Hall voltage signal under AC follows the equation,

$$
V_{H}=I_{o} R_{H}=V^{1 \omega} \sin (\omega t)-V^{2 \omega} \cos (2 \omega t)
$$

2

Although 1 st voltage has almost same signal at each $\phi$, 2nd voltage has completely different signals as shown Figure $4 \mathrm{~b}$ and Figure 4c. These results are come from the different contribution between DLT and FLT. The 2 nd order harmonic Hall voltage at each $\phi=0^{\circ}$ and $90^{\circ}$ with consideration of 2 nd order PMA energy follow the expression ${ }^{29}$,

$$
V_{x}^{1 \omega}=V_{y}^{1 \omega}=V_{A H E^{\cos } \theta_{M}}
$$

3

$$
V_{X}^{2 \omega}=\frac{V_{A H E}}{2}\left(A_{1} \Delta H_{D L}-B_{1} \Delta H_{F L}\right)
$$

$$
V_{y}^{2 \omega}=\frac{V_{A H E^{\cos } \theta_{M}}}{2}\left(B_{1} \Delta H_{D L}-A_{1} \Delta H_{F L}\right)
$$




$$
A_{1} \equiv \frac{\sin \theta_{M}}{H_{K, e f f} \cos 2 \theta_{M}-H_{K, 2} \sin \theta_{M} \sin 3 \theta_{M}+H_{e x t} \cos \left(\theta_{M}-\theta_{H}\right)}
$$

$$
B_{1} \equiv \frac{R \sin ^{2} \theta_{M}}{H_{e x t} \sin \theta_{H}}
$$

7

Following the Equation (2) to (7), we can rewrite DLT and FLT effective fields ( $\left.\Delta H_{D L}, \Delta H_{F L}\right)$ as function of $\theta_{M}$ from the measured harmonic Hall voltages as shown in Figure $4 \mathrm{~d}$ and Figure $4 \mathrm{e}$, respectively. Here, $\theta_{M}$ can be calculated using experimentally obtained the 1 st order harmonic Hall signal at each dose with Equation (3). The results show the different dose dependences on $\Delta H_{D L}$ and $\Delta H_{F L}$ with $\theta_{M}$. When the near of $\theta_{M}=15^{\circ}$, corresponding magnetization angle at external magnetic field of $3.1 \mathrm{kOe}$ in 0 ions $/ \mathrm{nm}^{2}, \Delta H_{D L}$ has small increasing tendency as shown in the inset in Figure $4 \mathrm{~d}$ but $\Delta H_{F L}$ has decreasing tendency at its magnitude according to dose amount. However, when $\theta_{M}>15^{\circ}$, both effective fields show great increase and complex behavior having a maximum peak point at $\theta_{M}$ of range from $40^{\circ}$ to $45^{\circ}$. According to simple macro-spin SOT mode ${ }^{29}$, there is no magnetization direction dependence on both effective SOT fields. However, there are much experimental evidences of the magnetization direction dependence on the effective SOT fields ${ }^{30,31,32}$. The higher order term of SOT can be one of the possible origins of complex angular dependence. According to Ref.30, the high order term of SOT is non-negligible and may cause complex angular dependence. In addition, if Helium ion irradiation modulates the higher-order term of the SOT just similar as the higher-order term of the PMA, the change in angular dependence can be estimated as a phenomenon caused by the Helium ion irradiation. Furthermore, another possible approach explaining such magnetization direction dependent effective SOT fields is from the framework of distorted Fermi surface ${ }^{33}$. The $\theta_{M}$ dependent effective fields in Figure $4 \mathrm{~d}$ and Figure $4 \mathrm{e}$ are rather complicated angular dependence compared with the theoretical results are based on the free-electron like model Hamiltonian with exchange coupling and Rashba effect. The experimental results reflect realistic band structures so that the more complex angular dependent explanation is acceptable. It is hard to analysis the exact origins separately. However, it is also true that the varying angular dependent effective fields by degree of ion irradiation has been experimentally observed as seen in Figure 4d and Figure 4e. And it is worth to note that if $H_{K, 2}$ is not considered in the calculation, the result has quite different tendency with Figure $4 \mathrm{~d}-4 \mathrm{e}$, suggesting the critical role of the 2 nd order anisotropy in precise analysis of harmonic Hall measurement in all range of $\theta_{M}$ (see the Supplementary Figure S5).

Because $\theta_{S H}$ is one of the most important material parameters for SOT based devices, understanding the correlation between ion irradiation induced $\theta_{S H}$ variation and $\mathrm{HM}$ layer state is important. In order to reveal the effect of the Helium ion irradiation on $\mathrm{HM}$ layer only, we irradiated the Helium ion on single $\mathrm{Pt}$ layer with thickness of $5 \mathrm{~nm}$ as same conditions introduced in sample fabrication description. We used 
the 4-probe measurement technique for measuring resistance with temperature range of $5 \mathrm{~K}$ to $225 \mathrm{~K}$ and calculated resistivity using sample geometry information with measured resistance. Here, the resistivity curve and the method of calculating resistivity at $300 \mathrm{~K}$ are explained in Supplementary Figure S6a. The resistivity of $\mathrm{Pt}\left(\rho_{P t}\right)$ at $300 \mathrm{~K}$ and $5 \mathrm{~K}$ is shown in Figure $5 \mathrm{a}$ and we can observe the increasing resistivity according to dose amount, 43.4 to $47.8 \mu \Omega \cdot \mathrm{cm}(109 \%)$ in $5 \mathrm{~K}$ and 56.8 to $60.9 \mu \Omega \cdot \mathrm{cm}(107 \%)$ in $300 \mathrm{~K}$ comparing 0 ions $/ \mathrm{nm}^{2}$ and 30 ions $/ \mathrm{nm}^{2}$. Figure $5 \mathrm{~b}$ displays the changed ratio of temperature coefficient $\left(a_{T e m p}\right)$, following $\rho=\rho_{0}\left(1+a_{T e m p} \bullet\left(T-T_{0}\right)\right.$ at linear resistivity increasing region $(T>50 \mathrm{~K})$, and residualresistivity ratio $(R R R)$, comparison of resistivities between $300 \mathrm{~K}$ and $5 \mathrm{~K}$ in here. We can find the decreasing tendency of $a_{T e m p}$ and $R R R$ both, it can be interpreted as increased influence of impurity at higher dose. Because the collision time is inversely proportional to the impurity density, decrease of $a_{T e m p}$ and $R R R$ value imply that the Helium ion irradiation makes extra scattering sources by structural distortion in Pt layer. The Figure $5 c$ shows the resistivity dependence of $\theta_{S H}$, and it can be calculated with $\Delta H_{D L}$ at each dose value using following equation ${ }^{34}$,

$$
\theta_{S H}=\frac{2 \mathrm{e}}{\text { ไhslash }} \frac{M_{S} t_{F} A_{H M}}{I_{0}} \Delta H_{D L}
$$

8

Here, $A_{H M}$ is the cross section area of flowing current into HM. We assume that the influence of irradiation on $M_{S}, t_{F}$ and $A_{H M}$ is small enough to ignore, because the irradiated dose amount is scarce to cause interlayer deformation ${ }^{19,20,27}$. So, we calculated the $\theta_{S H}$ with $M_{S}=1100 \mathrm{kA} \mathrm{m}^{-1}, t_{F}=0.8 \mathrm{~nm}, A_{H M}=10 \mu \mathrm{m}$ $\times 5 \mathrm{~nm}$ and $\Delta H_{D L}$ when $\theta_{M}=15^{\circ}$ at each dose amount using Equation (8). Error bar can be calculated as the averaged of values of the front and rear data starting from $\theta_{M}=15^{\circ}$. It is found that $\theta_{S H}$ has linear relation with increased resistivity by Helium ion irradiations, as well reported ${ }^{11,35,36}$. The $\theta_{S H}$ increases $^{2}$ 0.096 to 0.132 with resistivity growth from 56.8 to $60.9 \mu \Omega \bullet \mathrm{cm}$ in $300 \mathrm{~K}$, about 5 - 6 times greater than resistivity of bulk Pt $\left(10.6 \mu \Omega \cdot \mathrm{cm}\right.$ in $\left.20^{\circ} \mathrm{C}\right)$ in literature ${ }^{37}$. This result suggests that Helium ion irradiation process makes extra scattering sources, and they raise the resistivity of HM layer. And the extra scattering sources cause improvement of $\theta_{S H}$, resulting in more effective switching of the magnetization by SOT. Although the energy efficiency in view of operating the device is slightly worse due to the ion irradiation induced resistance increasement, the improved $\theta_{S H}$ ratio is $\sim 4$ times greater compared with resistivity increasement ratio. In terms of power $\left(P=R_{\text {sample }}{ }^{2}\right)$ consumption, the change in resistance and SHA has an inverse relationship. As a result, only $87.4 \%, 59.6 \%$, and $56.0 \%$ of power consumption is expected at 10,20 , and 30 ions $/ \mathrm{nm}^{2}$, respectively. (See Figure $5 \mathrm{~d}$ ) Here, the $R_{\text {sample }}$ and / are normalized resistance of $\mathrm{HM}$ and normalized current by $\theta_{S H}$ at each dose. Therefore, it means that Helium ion irradiation enables more efficient data writing in terms of energy consumption. And it is worth mentioning that the critical switching current equation shown in ref.28 does not match with our actual experimental value except only linear relationship with in-plane field. We expect because the formula is based on the macro spin model as like well-known Brown paradox ${ }^{38}$. Furthermore, there are reports that it does not match the 
actual value in the micron scale sample ${ }^{39,40}$. That's why we obtained $\theta_{S H}$ from the spin-orbit torque effective field measurement (see Figure 5c), not from the switching current density. Nevertheless, it is clear that the Helium ion irradiation leads to a decrease in the $H_{K, \text { eff }}$ an increase in the $\theta_{S H}$, and the more efficient the SOT induced magnetization switching.

In summary, we observe that Helium ion irradiation can properly reduce SOT induced switching current in $\mathrm{Pt}(5) / \mathrm{Co}(0.8) / \mathrm{MgO}(2)$ structure. The reduction appears $14.2 \%, 25.5 \%$, and $30.3 \%$ at dose of 10,20 , and 30 ions $/ \mathrm{nm}^{2}$ comparing with the pristine sample under the in-plane external magnetic field of $3.1 \mathrm{kOe}$. For understanding of physical reasons of decreasing tendency of the switching current, we considered two main possible origins of reduction, $H_{K, \text { eff }}$ and $\theta_{S H}$. From AHE measurement and GST method, we can extract $H_{K, \text { eff }}$ and it decreases from 13.7 to $8.5 \mathrm{kOe}(38.2 \%)$ comparing dose 0 and 30 ions $/ \mathrm{nm}^{2}$. Not only $H_{K, \text { eff }} \theta_{S H}$ also increase from 0.096 to 0.132 (27.2\%). Furthermore, it is revealed that improvement of $\theta_{S H}$ is consequence of increase of Pt resistivity by ion irradiation process. Although the power consumption is slightly worse due to the increase of the resistance, the decreased critical current caused by the improved $\theta_{S H}$ has a greater impact in power consumption. As a result, the ratio of power requiring for operation of device is calculated to consume only about $56.0 \%$ for switching at 30 ions $/ \mathrm{nm}^{2}$ compared to pristine sample, and this successful analysis on Helium ion irradiation induced modulation of SOT effect can be expected to improve efficiency of SOT based spintronic devices engineering.

\section{Methods}

\section{Thin film preparation and fabrication process}

The sample preparation process includes three steps, firstly Hall bar photolithography and deposition, secondly electrode fabrication, and Helium ion irradiation at last. This section will only explain up to step 2 , and step 3 will be explained in later paragraph (See the Helium ion microscope in Methods section). The sample is fabricated with lift-off process using photolithography and magnetron sputtering system. Normal metal layer and oxide layer is deposited using DC and AC power with stack of $\mathrm{Pt}(5) / \mathrm{Co}(0.8) / \mathrm{MgO}(2)$ on single surface polished $\mathrm{Si}$ substrate having $300 \mathrm{~nm}$ thickness $\mathrm{SiO}_{2}$ oxidated surface. The patterned Hall bar geometry consists of the current line of $10 \mu \mathrm{m}$ width and $40 \mu \mathrm{m}$ length and voltage line of $3 \mu \mathrm{m}$ width $16 \mu \mathrm{m}$ length. This geometry represses the offset error caused by sample shape. After deposition and patterning of sample, we deposit the electrode with stack of $\mathrm{Ta}(5) / \mathrm{Cu}(50)$ for electric measurement.

\section{Helium ion microscope}

HIM (Helium ion microscope, Carl Zeiss/ORION NanoFab), using high energy ionized Helium, is one of the brand-new microscopic techniques. Using HIM, we can obtain more detailed image in nano-scale structure compared with Gallium ion or electron based microscope owing to its penetration characteristic 
of high energy Helium ion 20,27 . But for utilizing HIM, we must make trimer, as the state of leaving only three atoms at the end of tip, stability and duration problems are remained. Even in such problems, the high penetration characteristic and low diffraction limitation of HIM come to be a big attraction. Because the HIM have low convergence angle, long best focus length and high penetration depth, more than few tens of nanometer scale, it also can be used as precise atomic structure destruction method. We expose the Helium ion with normal direction of sample plane with dose value of 0 to 30 ions $/ \mathrm{nm}^{2}$ at energy of 30 $\mathrm{keV}$ and current of $5.4 \sim 5.5 \mathrm{pA}$. The exposure process can be conducted from few hundred micron to few tens of nanometer size, and we conducted with area of $20 \mu \mathrm{m} \times 20 \mu \mathrm{m}$. The HIM is in the Central Core Research Facility center in DGIST, Korea.

\section{Transport measurement system with DC and AC current}

For measuring magnetic properties, we use the DC \& AC source (Keithley, 6221), nano-voltmeter (Keithley, 2182A), and lock-in amplifiers (Zurich Inst. SR830). Each equipment is connected to sample using customized PCB in the 2-axis rotational holder and the direction of magnetic field is controlled by rotating holder in prefer direction using motors. For measuring SOT induced magnetization switching, we use the current source for injecting pulse and nano-voltmeter for measuring Hall voltage. The magnetization is initialized to $+z$ direction (or $-z$ direction) using magnetic field and switching is induced under in-plane magnetic field using sweep mode of DC \& AC source. Comparing SOT induced magnetization switching measurement, the harmonic Hall measurement use different voltmeter, not nano-voltmeter but two lock-in amplifiers for detecting AC based Hall signals. One lock-in amplifier detects the 1st harmonic signal, and the other detects the 2 nd signal. All lock-in amplifiers are connected to sample with same voltage line, so the 1 st and 2 nd harmonic signal is measured simultaneously.

For temperature dependent measurement, we use the cryostat to be able to connect with nano-voltmeter and current source. The cryostat chamber also can be connected to customized PCB to instruments. The resistance is measured using 4-probe measurement with reading current of enough small current and sample atmosphere temperature can vary from $5 \mathrm{~K}$ to $275 \mathrm{~K}$ with gap of $0.25 \mathrm{~K}$ using chamber heater and Helium compressor system. Here, all current and voltage equipment is connected with sample using Bayonet Neill-Concelman cable for repressing noises.

\section{Declarations}

\section{Acknowledgements}

This work is supported by the National Research Foundations of Korea (NRF-2015M3D1A1070465, NRF2021R1A2C2007672, NRF-2020M3F3A2A02082437, NRF-2021M3F3A2A01037525, NRF2018R1A6A3A11041061). 


\section{Author contributions statement}

K.-S.L. and C.-Y.Y. conceived the projects; Sample fabrication was done by S.A. and J.-A.K; the measurements were performed by S.A., E.B.; Data analysis and manuscript preparation were done by S.A., K.-S.L., and C.-Y.Y.; The study was supervised by C.-Y.Y.

\section{Additional information}

The authors declare no competing interests.

\section{References}

1. Liu, L., Lee, O. J., Gudmundsen, T. J., Ralph, D. C. \& Buhrman, R. A. Current-Induced Switching of Perpendicular Magnetized Magnetic Layers Using Spin Torque from the Spin Hall Effect. Phys. Rev. Lett, 109, 096602 (2012).

2. Miron, I. M. et al. Perpendicular switching of a single ferromagnetic layer induced in-plane current injection. Nature, 476, 189-193 (2011).

3. Emori, S., Bauer, U., Ahn, S. M., Martinez, E. \& Beach, G. S. D. Current-driven dynamics of chiral ferromagnetic domain wall. Nat. Mater, 12, 611-616 (2013).

4. DC, M. et al. Room-temperature high spin-orbit torque due to quantum confinement in sputtered BixSe(1-x) films. Nat. Mater, 17, 800-807 (2018).

5. Ryu, K. S., Thomas, L., Yang, S. H. \& Parkin, S. Chiral spin torque at magnetic domain walls. Nat. Nanotechnol, 8, 527-533 (2013).

6. Liu, L. et al. Spin-torque switching with the giant spin Hall effect of tantalum., 336, 555-558 (2012).

7. Pai, C. F. et al. Spin transfer torque devices utilizing the giant spin Hall effect of tungsten. Appl. Phys. Lett, 101, 0122404 (2012).

8. Mosendz, O. et al. Quantifying Spin Hall Angles from Spin Pumping: Experiments and Theory. Phys. Rev. Lett, 104, 046601 (2010).

9. Liu, L., Moriyama, T., Ralph, D. C. \& Buhrman, R. A. Spin-Torque Ferromagnetic Resonance Induced by the Spin Hall Effect. Phys. Rev. Lett, 106, 036601 (2011).

10. Gweon, H. K., Lee, K. J. \& Lim, S. H. Influence of MgO Sputtering Power and Post annealing on Strength and Angular Dependence of Spin-Orbit Torques in Pt/Co/MgO Trilayers. Phy. Rev. Appl, 11, 014034 (2019).

11. Lee, J. W. et al. Enhanced spin-orbit torque by engineering Pt resistivity in Pt/Co/AlOx structures. Phys Rev. B, 96, 064405 (2017).

12. Pai, C. F. et al. Enhancement of perpendicular magnetic anisotropy and transmission of spin Hall effect induced spin currents by a $\mathrm{Hf}$ spacer layer in W/Hf/CoFeB/MgO layer structures. Appl. Phys. Lett, 104, 082407 (2014). 
13. Lee, H. Y. et al. Enhanced spin-orbit torque via interface engineering in $\mathrm{Pt} / \mathrm{CoFeB} / \mathrm{MgO}$ heterostructures. APL Mater, 7, 031110 (2019).

14. Cha, I. H., Kim, T., Kim, Y. J., Kim, G. W. \& Kim, Y. K. Thickness and composition-dependent spin-orbit torque behaviors in perpendicularly magnetized Ta/W (t)/CoFeB and Ta1-xWx/CoFeB junction structures. J. Alloy. Compd, 823, 153744 (2020).

15. Kim, Y. J. et al. Large reduction in switching current driven by spin-orbit torque in W/CoFeB heterostructures with W-N interfacial layers. Acta Mater, 200, 551-558 (2020).

16. Hasegawa, K., Hibino, Y., Suzuki, M., Koyama, T. \& Chiba, D. Enhancement of spin-orbit torque by inserting CoOx layer into Co/Pt interface. Phys. Rev. B, 98, 020405 (2018).

17. Yun, J. et al. Lowering critical current density for spin-orbit torque induced magnetization switching by ion irradiation. Appl. Phys. Lett, 115, 032404 (2019).

18. Dunne, P. et al. Helium Ion Microscopy for Reduced Spin Orbit Torque Switching Currents. Nano. Lett, 20, 7036-7042 (2020).

19. Fassbender, J., Ravelosona, D. \& Samson, Y. J. Tailoring magnetism by light-ion irradiation. Phys. D: Appl. Phys, 37, R179-R196 (2004).

20. Hlawacek, G., Veligura, V., van Gastel, R. \& Poelsema, B. Helium ion microscopy. J. Vac. Sci. Technol. $B, 32,020801$ (2014).

21. Chappert, C. et al. Planar patterned magnetic media obtained by ion irradiation. SCIENCE, 280, 19191922 (1998).

22. Franken, J. H. et al. Precise control of domain wall injection and pinning using helium and gallium focused ion beams. J. Appl. Phys, 109, 07504 (2011).

23. Nembach, H. T. et al. Tuning of the Dzyaloshinskii-Moriya interaction by He+ ion irradiation. arXiv:2008.06762(2020).

24. Juge, R. et al. Helium ions put magnetic skyrmions on the track. Nano Lett, 21, 2989-2996 (2021).

25. Zhao, X. et al. Enhancing domain wall velocity through interface intermixing in W-CoFeB-MgO films with perpendicular anisotropy. Appl. Phys. Lett, 115, 122404 (2019).

26. Zhao, X. et al. Spin-orbit torque driven multi-level switching in He+ irradiated W-CoFeB-MgO Hall bar with perpendicular anisotropy. Appl. Phys. Lett, 116, 242401 (2020).

27. Livengood, R., Tan, S., Greenzweig, Y., Notte, J. \& McVey, S. Subsurface damage from helium ions as a function of dose, beam energy, and dose rate. J. Vac. Sci. Technol. B, 27, 3244 (2009).

28. Lee, K. S., Lee, S. W., Min, B. C. \& Lee, K. -J. Threshold current for switching of a perpendicular magnetic layer induced by spin Hall effect. Appl. Phys. Lett, 102, 112410 (2013).

29. Yun, S. J. et al. Accurate analysis of harmonic Hall voltage measurement for spin-orbit torques. NPG Asia Materials, 9, e449 (2017).

30. Garello, K. et al. Symmetry and magnitude of spin-orbit torques in ferromagnetic heterostructures. Nat. Nanotechnol, 8, 587-593 (2013). 
31. Park, E. S., Lee, D. K., Min, B. C. \& Lee, K. -J. Elimination of thermoelectric artifacts in the harmonic Hall measurement of spin-orbit torque. Phys. Rev. B, 100, 214438 (2019).

32. Qiu, X. et al. Angular and temperature dependence of current induced spin-orbit effective fields in $\mathrm{Ta} / \mathrm{CoFeB} / \mathrm{MgO}$ nanowires. Sci. Rep, 4, 4491 (2014).

33. Lee, K. S. et al. Angular dependence of spin-orbit spin-transfer torques. Phys. Rev. B, 91, 144401 (2015).

34. Khvalkovskiy, A. V. et al. Matching domain-wall configuration and spin-orbit torques for efficient domain-wall motion. Phys. Rev. B 87, 020402(R)(2013).

35. Sagasta, E. et al. Unveiling the mechanisms of the spin Hall effect in Ta. Phys. Rev. B, 98, 060410 (2018).

36. Nakagawara, K. et al. Temperature-dependent spin Hall effect tunneling spectroscopy in platinum. Appl. Phys. Lett, 115, 162403 (2019).

37. Serway, R. A. Principle of Physics 2nd edn (Fort Worth, Texas, USA, 1999).

38. BrownJr. W. F. Micromagnetics (Wiley, New York, USA, 1963).

39. Ranjbar, R., Suzuki, K. Z., Sasaki, Y., Bainsla, L. \& Mizukami, S. Current-induced spin-orbit torque magnetization switching in a MnGa/Pt film with a perpendicular magnetic anisotropy. Jpn. J. Appl. Phys, 55, 120302 (2016).

40. Neumann, L. et al. Temperature dependence of the spin Hall angle and switching current in the ncW(0)/CoFeB/MgO system with perpendicular magnetic anisotropy. Appl. Phys. Lett, 109, 142405 (2016).

\section{Figures}


(a)
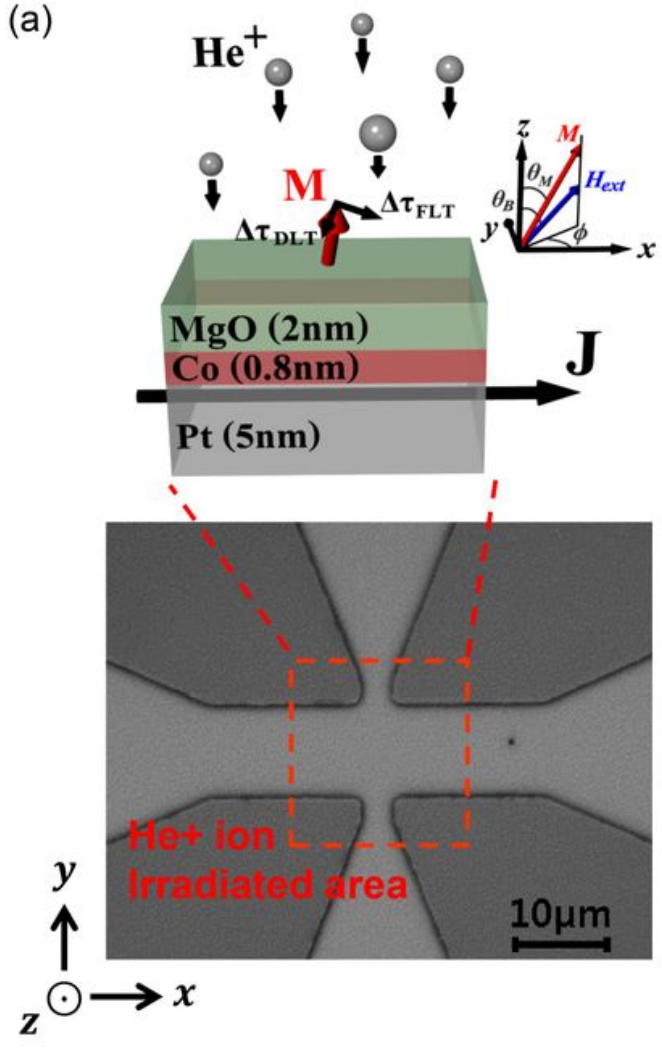

(b)

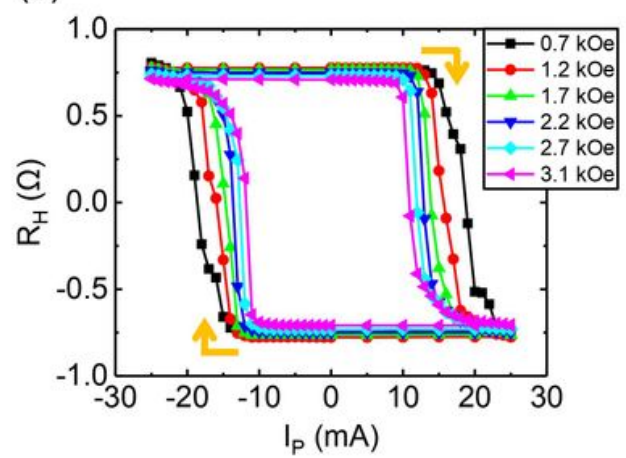

(d)

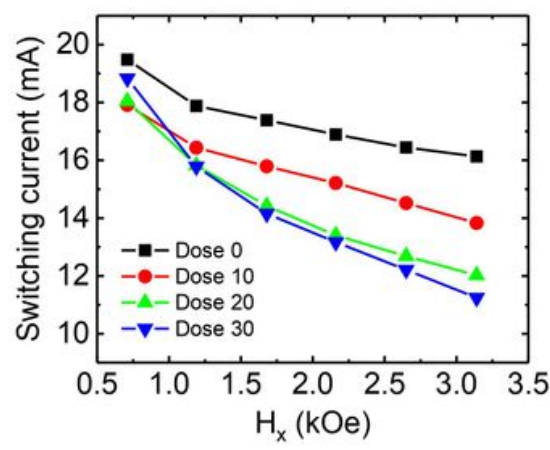

(c)

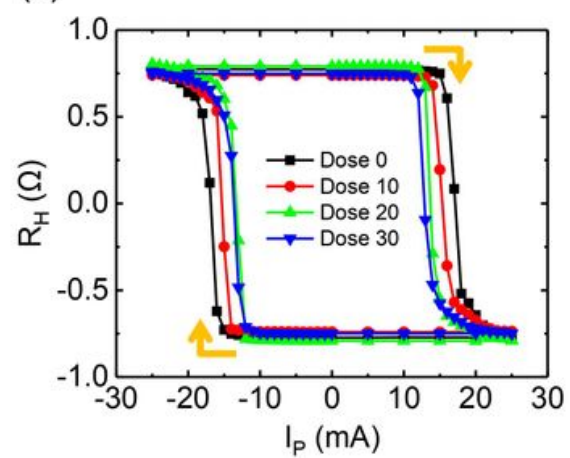

(e)

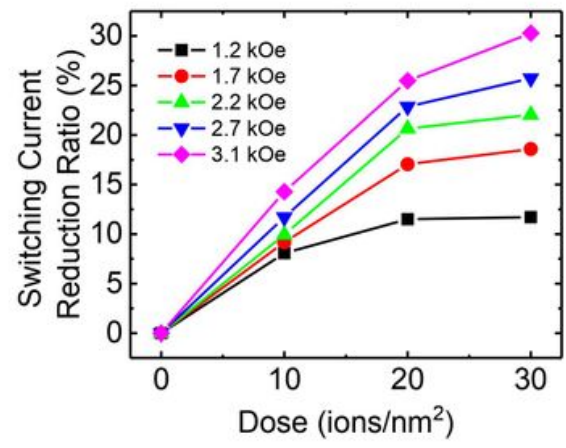

Figure 1

Please see the Manuscript file for the complete figure caption 
(a)

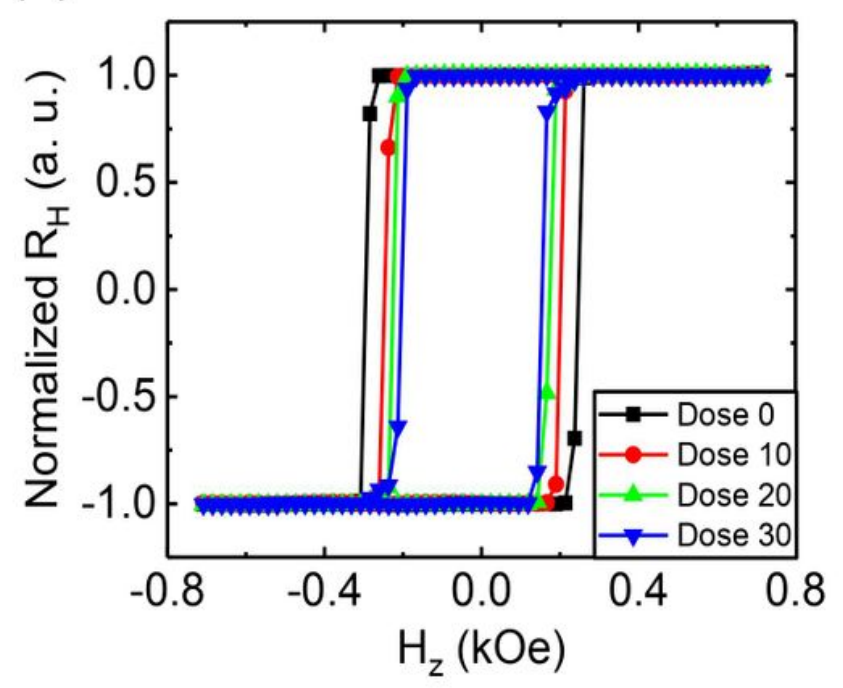

(c)

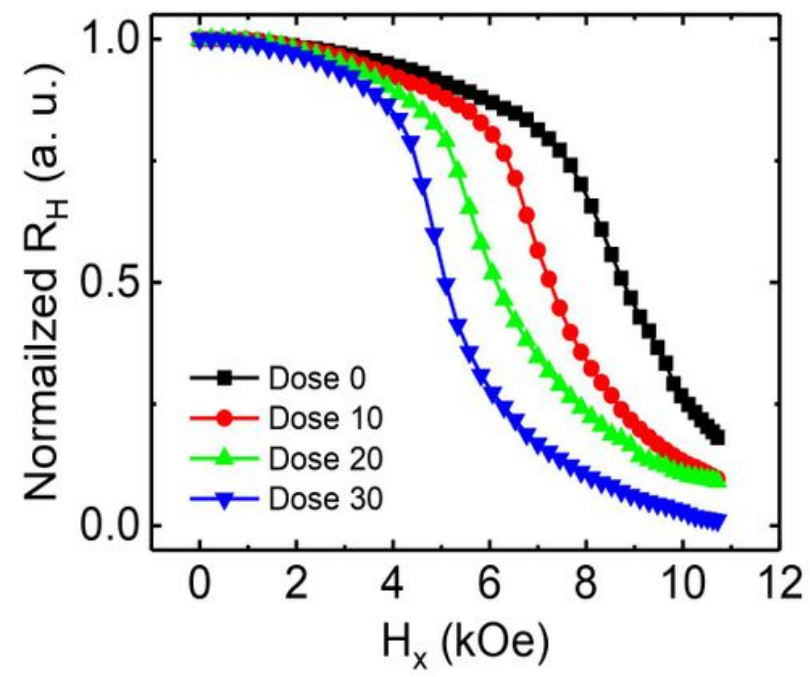

(b)

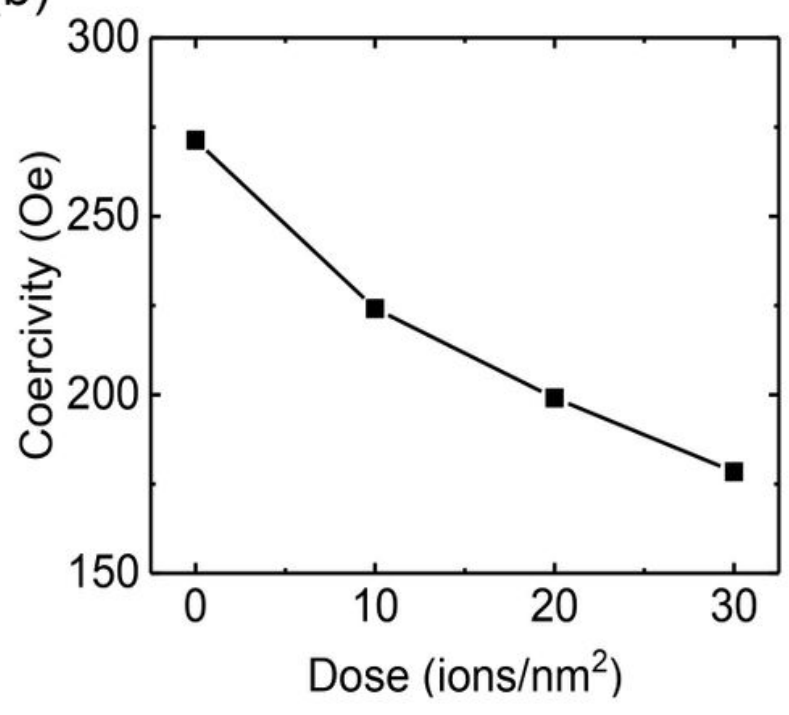

(d)

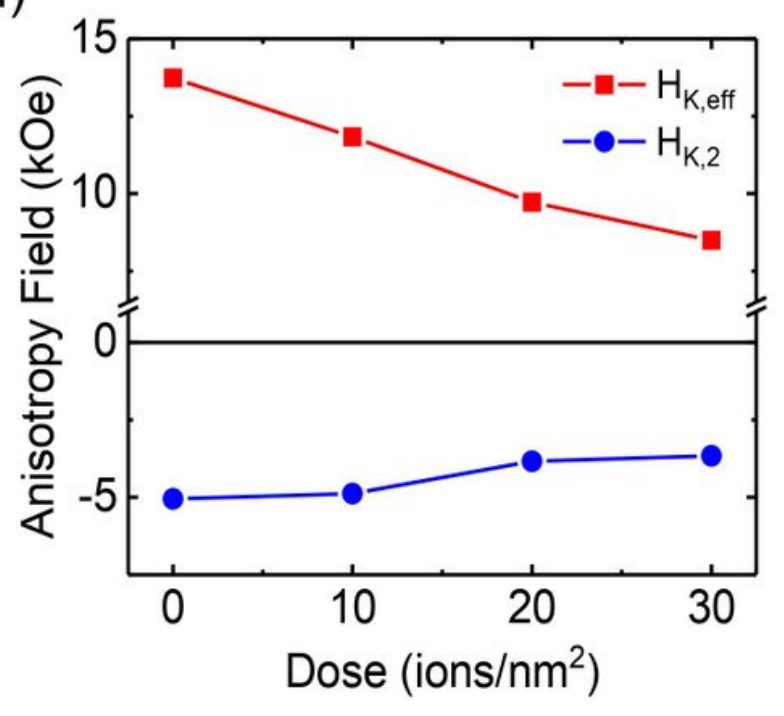

Figure 2

AHE measurement results for $\mathrm{Pt}(5) / \mathrm{Co}(0.8) / \mathrm{MgO}(2 \mathrm{~nm})$ samples ay various Helium ion irradiation doses amount. (a) Normalized AHE loops with the external magnetic field perpendicular $(\mathrm{Hz})$ for various doses. (b) The coercivities from the AHE loops as a function of doses. (c) Normalized AHE signals of various doses with the in-plane field $(\mathrm{Hx})$ for GST method. Here, the solid lines are trending lines. (d) The 1st and 2nd anisotropy fields (HK,eff, HK,2) extracted by the GST method are plotted for various doses. We measured all AHE with the reading current of $100 \mu \mathrm{A}$ for various doses of $0,10,20$, and $30 \mathrm{ions} / \mathrm{nm} 2$. Current flow along $\mathrm{x}$-axis and Hall voltage is measured in $\mathrm{y}$-axis. 
(a)

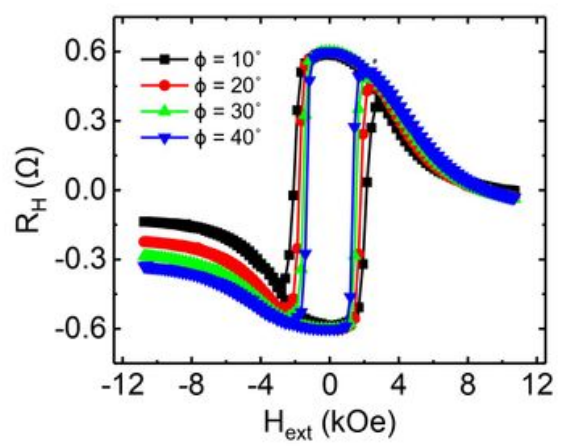

(d)

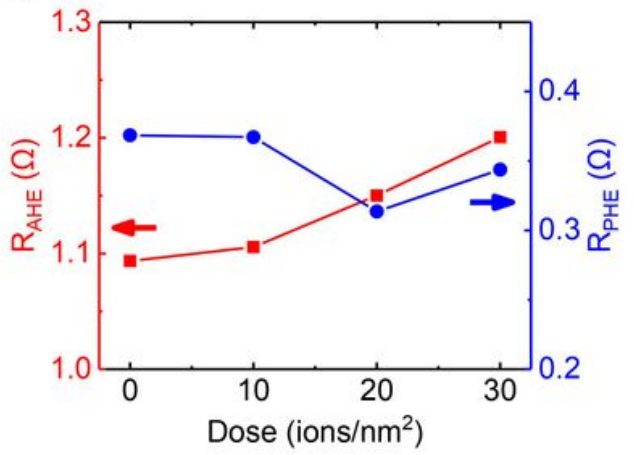

(b)

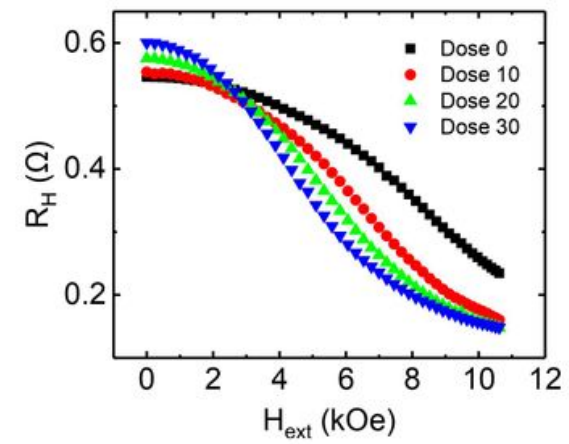

(e)

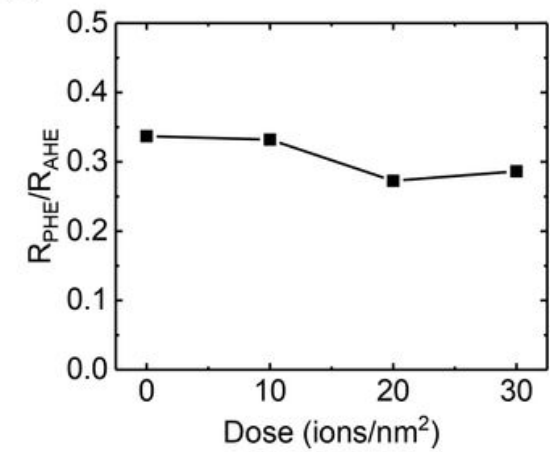

(c)

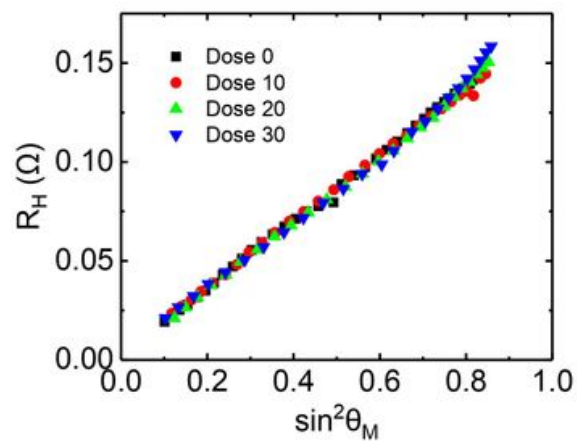

Figure 3

AHE and PHE resistances measured for various doses. (a) Hall resistance loops of dose amount of 30 ions $/ \mathrm{nm} 2$ in $\phi=10^{\circ}-40^{\circ}$. The oblique magnetic field $\left(\theta \mathrm{B}=80^{\circ}\right)$ is applied to obtained mixed Hall resistances signals. (b) AHE contribution and (c) $\mathrm{RH}$ as a function of $\sin 2 \mathrm{\theta M}$ to extract the PHE contribution from the slopes of those plots. The measurements results are obtained for various doses at $\phi=40^{\circ}$ at $\theta B=80^{\circ}$. (d) Resulting RAHE and RPHE values and (e) its ratio, RPHE/RAHE, at each dose. 
(a)

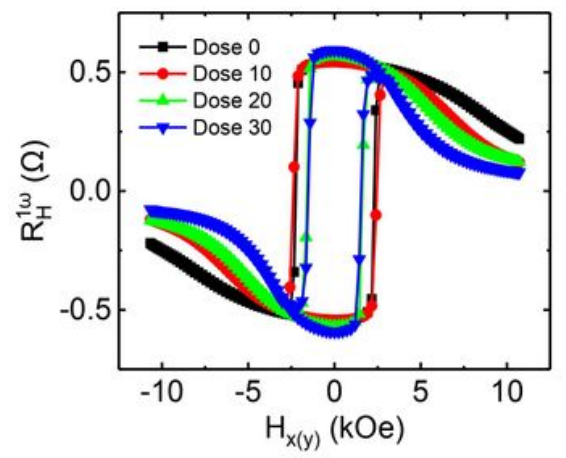

(d)

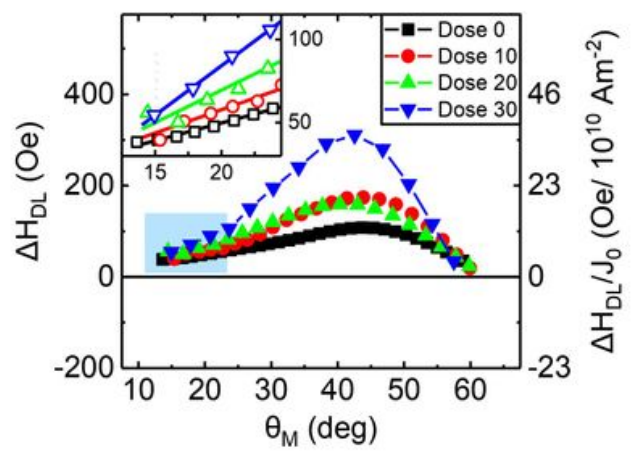

(b)

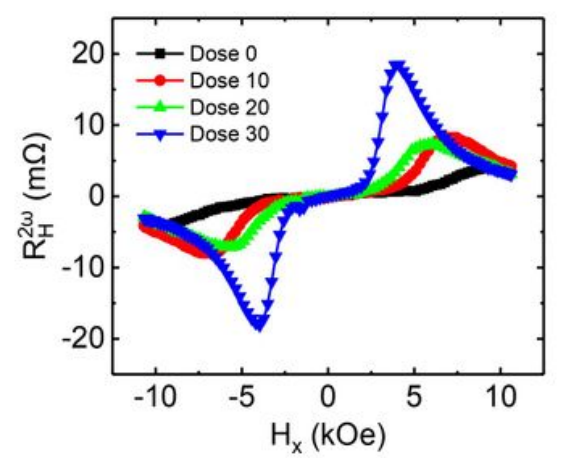

(e)

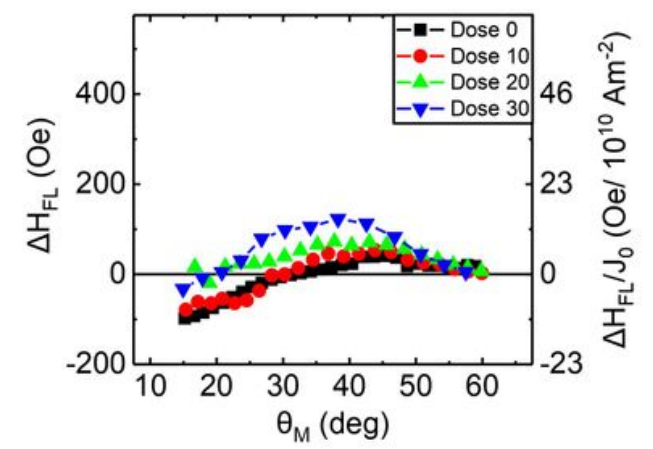

(c)

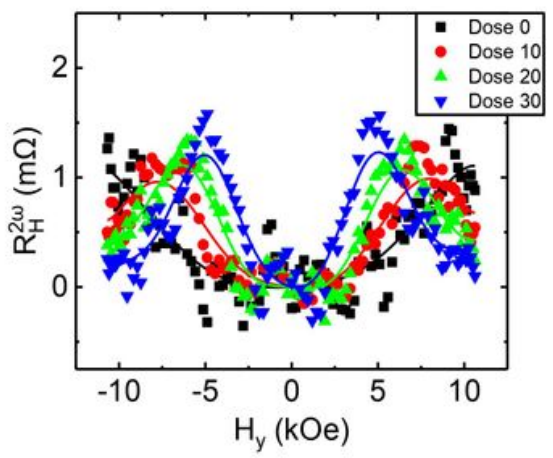

Figure 4

Please see the Manuscript file for the complete figure caption 
(a)

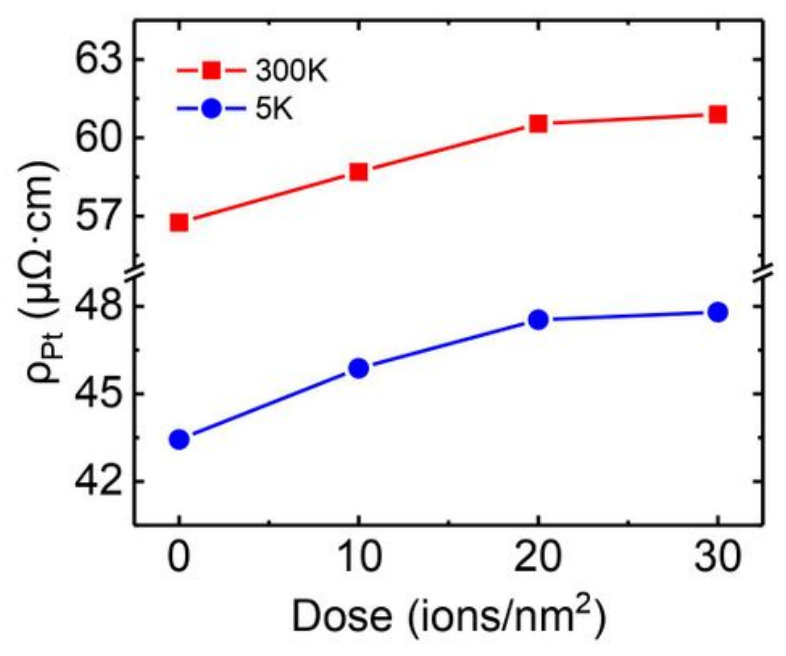

(c)

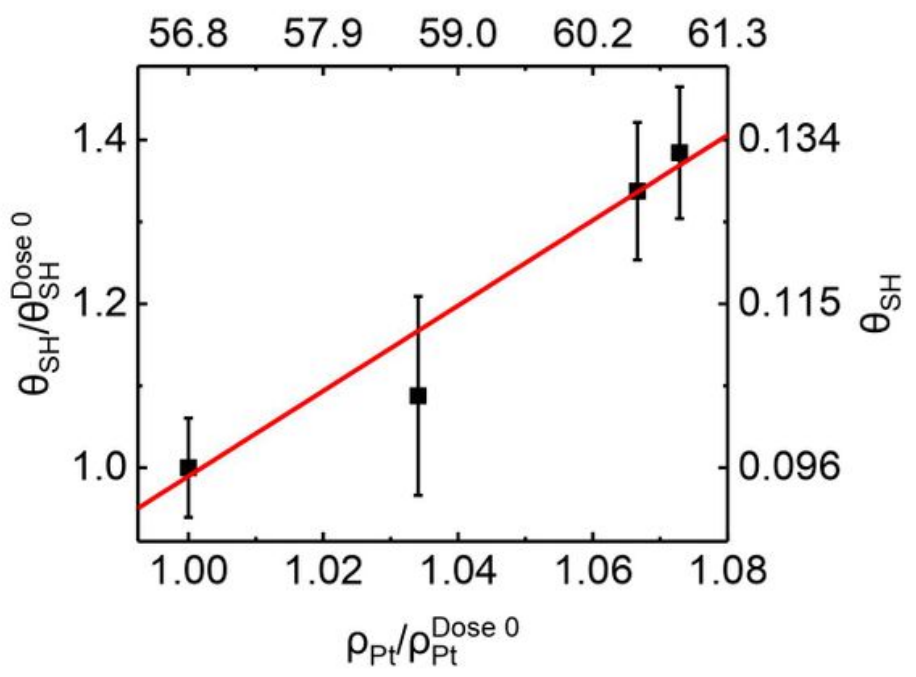

(b)

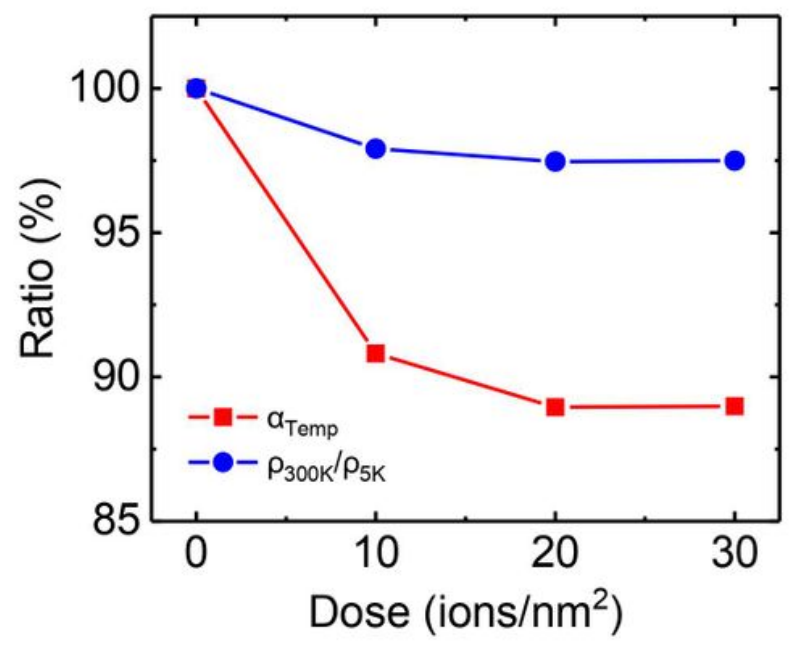

(d)

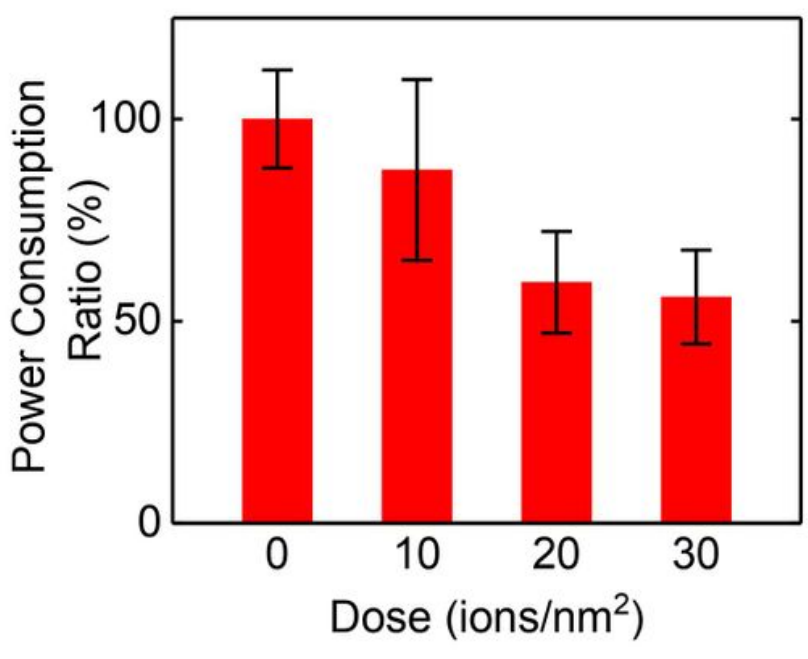

Figure 5

Resistivity changes of Platinum single layer for various doses and its comparison with SHA. (a) Measured Platinum single layer resistivity at $300 \mathrm{~K}$ and $5 \mathrm{~K}$ as function of dose amounts. (b) The ratios of temperature coefficient aTemp and RRR comparing to the pristine sample values (c) Comparison of $\theta \mathrm{SH}$ and resistivities at $300 \mathrm{~K}$ for various doses. Red solid line is linear fitted line of $\theta \mathrm{SH}$ with platinum resistivity. (d) Calculated power consumption ratio at each dose comparing pristine sample.

\section{Supplementary Files}

This is a list of supplementary files associated with this preprint. Click to download.

- Supplementarylnformation.pdf 